\title{
Le bénévolat de compétences comme forme de résistance des seniors
}

\author{
Étude de cas de l'association ECTI - professionnels \\ bénévoles seniors ${ }^{1}$
}

\author{
Soukey Ndoye, \\ Doctorante en sociologie, EA Éthique, politique et Santé \\ Université Paris Descartes (France)
}

\begin{abstract}
[Résumé] Basé sur une enquête menée auprès de responsables bénévoles, cet article décrit les manifestations du bénévolat de compétences des seniors et interroge ses enjeux en prenant le cas particulier de l'association Ecti. II montre comment cette forme de bénévolat s'inscrit dans une dynamique de professionnalisation des activités bénévoles et comment elle est traversée par des tensions autour d'une quête de valorisation économique de ces activités. II montre enfin comment des obstacles tels que les réticences des consultants à l'égard du "bénévolat de compétences » s'opposent à ce projet et créent les conditions d'installation d'une forme particulière de résistance.
\end{abstract}

Mots-clés : bénévolat, compétences, professionnalisation, résistance, retraités

[Abstract] Based on an investigation led with voluntary people in charge, this article describes the appearance of the "elder skills-based volunteering " and questions its issues through the specific case of the association Ecti. It shows how this form of volunteering engagement joins a dynamic of professionnalization of the volunteering activities and how it is crossed by tensions around a search for economic valuation of such activities. It finally shows how obstacles such as the critics of the consultants towards « elder skills-based volunteering » oppose this project and create the conditions of the emergence of a particular form of resistance.

Keywords: volunteering, skills, professionalization, resistance, retired people

$\mathrm{D}^{2}$ ans ses usages sociaux, l'expression «bénévolat de compétences » renvoie généralement à une forme d'engagement bénévole visant à assurer le transfert de compétences des salariés de l'entreprise vers des structures associatives (Baculard 2006; Bory 2013). Cette définition en fait une notion non seulement indexée au seul groupe d'âge des actifs en âge de travailler, mais aussi à l'unique monde social de l'entreprise. Or, à l'épreuve de la retraite, une frange importante de la population de seniors réinvestit leurs ressources et compétences (professionnelles et extraprofes-

1 Les termes seniors et retraités sont ici employés de manière indistincte pour désigner les individus sortis définitivement du marché de l'emploi du fait de leur âge : qu'ils aient été bénéficiaires des systèmes de préretraite, qu'ils soient au chômage en fin de carrière ou qu'ils soient déjà pensionnés d'un régime de retraite. 
sionnelles) dans le secteur associatif en vue d’y mener une activité bénévole (Guillemard 2010 ; Viriot Durandal 2003).

Dans cet article, l'expression «bénévolat de compétences » désignera un travail non marchand (Simonet-Cusset 2004), un acte gratuit, librement consenti et destiné à autrui (Ferrand-Bechman 1992 ; Godbout 1994; Gagnon et Fortin 2002; Archambault 2005) qui comporte trois dimensions principales. C’est un bénévolat qui prend place dans le cadre d'un engagement social structuré au sein d'une organisation non marchande (Viriot Durandal et Reguer 2011; Bernardeau-Moreau et Hély 2007). Il mobilise des ressources issues des milieux professionnels et des réseaux d'acteurs autour d'objectifs communs (Piris et Dupuy 2007). Il est aussi susceptible de transformer l'environnement social, économique et politique des acteurs (Zarifian 2001). Si cette définition restreint les formes multiples de l'engagement social à la seule expression du bénévolat formel, elle couvre néanmoins le registre de participation bénévole de l'ensemble des groupes sociaux.

La démarche empruntée ici consiste à réinterroger l'engagement bénévole des seniors à l'aune de la rencontre de deux mouvements : d'une part, la professionnalisation du secteur associatif qui mobilise les compétences des bénévoles ainsi que des logiques gestionnaires calquées sur le modèle de l'entreprise et du travail marchand (Laville 2003 : 89); d'autre part, la "police des âges » qui contingente les rôles et les statuts politiques, sociaux et économiques des acteurs, et qui entraîne une perte de valeurs ou une démonétisation des ressources pour certains retraités (Percheron et Rémond 1991; Viriot Durandal 2003). Dès lors, le bénévolat de compétences est-il une quête de reconnaissance et de valorisation d'un nouveau statut social des seniors ? Est-il pour eux un moyen de résister à un système social qui participe à leur exclusion du monde productif?

Partant d'une analyse des stratégies collectives développées au niveau d'une association particulière, nous défendons l'hypothèse que le bénévolat de compétences des seniors se situe au carrefour de deux mouvements de contestation rattachés à une même catégorie d'âge. D'un côté, une "retraite-revendication» qui dénonce l'inadéquation entre les ressources et les statuts, entre les potentiels et les biens individuels ou collectifs des retraités (Guillemard 1972 ; Viriot Durandal 2003). Puis de l'autre, une "retraite inclusion » qui réclame le droit à l'emploi des travailleurs âgés (Poussou-Plesse 2007). Le bénévolat de compétences des seniors renverrait alors à la quête d'un statut intermédiaire situé à l'intersection du statut antérieur de professionnel et de celui, actuel, de bénévole. Il constituerait aussi, en creux, une dénonciation de la centralité du travail dans nos sociétés. 
Pour défendre cet argument, notre démarche s'appuie sur une étude de cas monographique de l'association Ecti ${ }^{2}$. Cette méthode a été particulièrement féconde pour englober plusieurs dimensions de l'association, qu'ils s'agissent des expériences individuelles, des logiques organisationnelles ou des stratégies développées. C'est à partir de l'expérience et du discours de 15 responsables bénévoles de l'association que nous proposons une analyse des dynamiques individuelles et collectives du bénévolat de compétences des seniors. Parce que ces responsables participent directement à l'action en accomplissant des missions bénévoles sur le terrain, ou indirectement en prenant part aux processus d'orientation stratégique de l'association, ce choix s'est révélé particulièrement pertinent. L'enquête a été menée en 2010 puis reconduite $2013^{3}$.

Comprendre comment le bénévolat de compétences peut être à l'origine de l'émergence d'une forme de résistance particulière des seniors (3) suppose de décrire au préalable les contours de cette forme bénévolat (1) puis de mettre en lumière les contradictions qui alimentent les suspicions à l'égard de ce bénévolat et les réponses qu'elles engendrent en retour (2).

\section{Le bénévolat de compétences des seniors : forme de professionnalisation de l'action bénévole}

La professionnalisation de l'activité bénévole au sein d'Ecti conjugue deux types de logiques : d'une part, celle qui tend à structurer l'association autour du modèle d'une entreprise classique ; d'autre part, celle qui pose un filtre hautement sélectif à l'adhésion bénévole au sein de l'association dans l'optique de constituer un groupe de seniors, experts dans leur domaine professionnel, mais socialement homogène.

\subsection{Une organisation comparable à celle d'une grande entreprise}

L'association Ecti a son siège au cœur d'un microcosme d'entreprises, dans un immeuble situé en pleine zone d'activités dans la banlieue parisienne. Le 4e étage s'ouvre sur un espace d'une centaine de mètres carrés dédié à l'association. Il abrite

2 L'association Échanges et consultations techniques internationales (Ecti) a été fondée en 1974 à l'initiative de Paul Borel, alors retraité des Nations Unies. En 2007, l'association adopte une nouvelle dénomination mieux en phase avec ses présentes préoccupations : Ecti - professionnels seniors. L'acronyme Ecti désigne aujourd'hui Entreprise, collectivité territoriale, insertion. Nous continuerons néanmoins à utiliser dans cet article à utiliser le terme court Ecti pour désigner l'association Ecti - professionnels bénévoles.

3 Sur cet échantillon de 15 personnes, 11 responsables ont été interrogés en 2010, dont 7 ont été réinterrogés en 2013. 4 responsables ont rejoint l'échantillon la même année. Et on compte 2 départs de l'association et 1 décès. Le plus jeune a 56 ans, le plus âgé 85 ans. 
des salles de réunion ainsi que de grands bureaux dont l'agencement et l'occupation rappellent le modèle des open space. Aux premiers abords, règne une atmosphère d'effervescence due à une activité incessante de va-et-vient, de discussions autour d'un café ou d'une grande photocopieuse. On y observe un défilé de seniors (majoritairement des hommes en costume et cravate) qui interagissent ou échangent avec les deux assistantes postées à l'accueil, qui viennent faire des photocopies, ou qui occupent les sièges d'attente dans un coin de l'espace.

À son origine, l'association était vouée à apporter une assistance technique aux pays en développement par la transmission de compétences, dans le cadre de missions à visée principalement économique. Progressivement, Ecti s'est tournée vers le territoire national français où elle effectue désormais deux tiers de ses interventions. Celles-ci sont de quatre ordres et ciblent autant de publics. Les missions à visée sociale sont essentiellement destinées à l'insertion sociale et professionnelle des publics comme les détenus en fin de peine et les demandeurs d'emploi. Auprès des Écoles et des universités, l'association tente de participer à la formation et à l'insertion professionnelle des jeunes en développant des actions de parrainage entre autres. Une catégorie de missions est spécifiquement dédiée aux conseils en direction des collectivités territoriales ; l'association s'est notamment spécialisée dans le domaine de la prévention des risques professionnels. Les missions de conseils en création, cessation et reprise d'entreprises s'adressent principalement aux PME, TPE et PMI. Toutefois, cette dernière catégorie d'interventions implique que les bénévoles d'Ecti investissent les mêmes terrains et missions que certains consultants actifs sur le marché du travail. Nous verrons plus loin comment cette collision est source de tensions.

Aujourd'hui, Ecti compte entre 2500 et 3000 bénévoles ${ }^{4}$. Son modèle économique est fondé sur l'adhésion d'organismes publics et privés qui apportent une cotisation en échange de services gratuits (les missions) délivrés par des bénévoles qui, eux aussi, apportent une cotisation.

Pour gérer ses différentes missions, l'association s'est peu à peu structurée. Elle compte 22 délégations régionales et une entité dans pratiquement $90 \%$ des départements français. Le siège recouvre les services centraux où l'on retrouve, entre autres, un service de gestion des ressources humaines bénévoles. La structuration et le mode de fonctionnement de ce service (en sous-entités affiliées aux différentes spécialités et domaines d'expertise) permettent de le comparer au département GRH d'une grande entreprise.

Outre le fait que tous les adhérents de l'association sont d'emblée considérés comme des bénévoles, un certain nombre d'entre eux assurent des responsabilités au sein de la structure. Ces bénévoles responsables assurent la majorité des charges ad-

4 II s'agit là d'une estimation délivrée par l'association, car aucune donnée statistique n'est disponible à ce jour. 
ministratives de l'association tout en les cumulant, pour quelques-uns, avec des missions d'expertise sur le terrain. Néanmoins ces responsables peuvent s'appuyer sur les 6 salariés du siège qui s'occupent des fonctions supports tels que la réception et le secrétariat, la comptabilité et les services généraux.

\subsection{De l'expertise professionnelle au bénévolat senior d'expertise}

L'engagement bénévole au sein d'Ecti révèle un groupe social fortement homogène du point de vue socio-économique, dont la reproduction est assurée par un mode de recrutement qui se structure autour de mécanismes de sélection et, implicitement, d'exclusion.

Le principal contingent des bénévoles de l'association est constitué d'anciens cadres supérieurs diplômés de grandes écoles 5 . Cette sélection de hauts profils laisse peu de place aux autres candidats. Mais surtout, elle sous-tend une volonté de constituer un groupe de pairs voulant préserver un statut socialement valorisant ${ }^{6}$, comme en témoigne l'expérience de Sophie, une cadre supérieure dans le domaine bancaire au parcours professionnel entrecoupé de ruptures qui finit par subir un déclassement en fin de carrière : « J'ai décidé de venir chez Ecti, car les autres associations ne me convenaient pas. En fait l'association est composée d'une majorité de cadres et donc, les missions sont plus des missions de cadres que des missions de travail opérationnel ».

Cette tendance à l'homogénéisation des profils constitue le socle d'une stratégie de sélection essentiellement tournée vers le recrutement de seniors particulièrement dotés en compétences, et dont l'expertise sur un domaine ou un métier est mesurée, $a$ priori, à l'aune du parcours professionnel accompli, puis a posteriori, dans le cadre des missions qui sont confiées. C'est le cas de Jean, ce diplômé d'un CAP d'ajusteur qui a terminé sa carrière comme ingénieur au bureau d'études d'une grande firme automobile française. Deux ans après son arrivée à Ecti, il est le bénévole qui a effectué le plus de missions, refusant même parfois celles qui ne lui semblent pas correspondre à son domaine de compétences : «il ne faut pas faire n'importe quoi, car sinon c'est le demandeur qui en pâtit. Si on ne peut pas, on sollicite un autre expert ». Cette position exigeante vis-à-vis de l'intervenant expert rejoint celle communément adoptée au sein de l'association et expressément stipulée dans le projet associatif : « les adhérents d’Ecti s'engagent à intervenir avec professionnalisme ».

5 La plaquette de présentation de l'association dénommée « Des compétences au service du développement économique et social en France et à l'International », indique que $38 \%$ des membres de l'association sont issus de formations scientifique et technique, $27 \%$ des filières de droit, gestion et sciences économiques, $12 \%$ ont été en École de commerce, $13 \%$ ont reçu une formation professionnelle. En 2013, le site Internet de l'association indique des données identiques : www.ecti.org.

6 Afin de garantir l'anonymat des personnes interviewées, leurs prénoms ont été modifiés. 
Outre le premier niveau de recrutement qui permet d'intégrer de nouveaux bénévoles, le choix des responsables de l'association requiert également une attention particulière. La disponibilité d'un carnet d'adresses encore mobilisable est un argument décisif lorsqu'il s'agit de prendre des responsabilités dans l'association. Le responsable doit être capable de faire connaître l'organisation en incarnant ses valeurs auprès d'un réseau de pairs encore actifs sur le marché du travail et susceptibles de rejoindre ses rangs. De plus, il doit avoir une envergure relationnelle qui lui permettra de dénicher des missions pour l'association. Ainsi en est-il de Marc, ancien cadre supérieur de l'industrie agroalimentaire, qui a pris la responsabilité de cette spécialité quatre mois après son arrivée chez Ecti. Ayant travaillé essentiellement dans une région française durant sa vie professionnelle, il y a développé un réseau relationnel important qu'il décide d'ouvrir à la délégation Ecti située dans la région : « Comme je connais beaucoup de monde là-bas, on essaie d'utiliser tout cela pour trouver des missions. J'utilise tout simplement mon carnet d'adresses ».

\section{Une activité bénévole à valeur économique ou le paradoxe d'Ecti}

Dès lors qu'il tend à outrepasser les finalités traditionnellement non marchandes de l'activité associative, Ecti subit de fortes pressions générées par les réticences des pouvoirs publics et de certains acteurs du marché du travail comme les consultants à l'égard du bénévolat de compétences des seniors. Ceux-ci regardent d'un œil inquiet le secteur du conseil en entreprises investi par des travailleurs bénévoles. Une compréhension fine de ces crispations passe par une analyse des modalités de la pratique du bénévolat au sein d'Ecti et des transformations intervenues dans la stratégie de l’organisation depuis le début des années 2000.

\subsection{Une association qui évolue au prime abord dans le sillage d'une activité marchande...}

À l'origine, l'action bénévole au sein d'Ecti avait une visée humanitaire et était destinée à l'aide au développement. Mais l'association opère un virage stratégique conséquent dès le début des années 2000 en glissant d'une logique « d'assistance » vers une logique de "production ». Cette évolution se concrétise lorsque l'association développe un kit destiné à accompagner les collectivités territoriales dans l'adoption du Document unique d'évaluation des risques professionnels 7 . Ecti devient avec cet outil la première association qui propose aux collectivités ce qui est dès lors désigné

7 Le Document unique a été créé par décret n²001-1016 du 5 novembre 2001 transposant la directive européenne sur la prévention des risques professionnels. 
comme un "produit » et qui s'apparente à « un service marketing » selon un responsable. L'association s'inscrit dans une logique de prestation commerciale qui se traduit par la création d'un nouveau poste de responsabilité : le « chef de produit».

La transition vers une logique de production s'ancre ainsi dans l'usage voire l'institutionnalisation d'un registre sémantique emprunté au champ des activités marchandes. Mais ce glissement fait débat à l'intérieur même de l'association. En témoignent les discours contradictoires de deux responsables interrogés sur le rôle clé des bénévoles chargés de dénicher des missions pour l'association. Si pour l'un, les frontières entre le monde associatif et l'entreprise sont bien définies, pour l'autre, elles sont ténues. Le premier, Michel, tente de rectifier son emprunt du mot «client» pour nommer les commanditaires de ces missions : "Ils vont essayer de faire venir des clients à l'Ecti. Enfin je dis clients, mais on n'appelle pas ça des clients, ce sont de nouveaux demandeurs de missions ». Le second, Guy, légitime son emploi en confondant les enjeux de l'association à ceux d'une entreprise classique : «Qu'est-ce que c'est qu'une entreprise ? Elle fait vivre des gens. C'est ce qu'on fait. La seule différence c'est que nous, on ne cherche pas de bénéfice à la fin de l'année comme le souhaite l'entreprise. Autrement, on fait pareil, on cherche des clients, on négocie la cotisation, on monte des missions pour eux ».

Derrière ce mouvement de glissement qui s’inscrit principalement dans la logique de professionnalisation, on perçoit une interrogation sur la finalité et la valeur de l'activité bénévole, précisément sur le rôle des retraités bénévoles dans la société. Ce qui pose question ici n'est pas tant la valeur symbolique de l'engagement que sa valeur économique. Pour autant, malgré le fait que cette quête n'est pas nécessairement partagée par l'ensemble des responsables bénévoles, on peut noter une tendance quasi spontanée des bénévoles à pointer l'efficacité et même le poids économique de leur action. Les propos tenus par Pierre Chéron le 14 avril 2013 dans le journal de 20 heures d'une grande chaîne de télévision française en témoignent. Ce bénévole senior, responsable de la délégation Ecti de la Manche, déclarait de façon péremptoire : « On va contribuer à l'expansion économique, à la création d'emploi, à la cohésion sociale, donc pour nous c'est fabuleux!»

\section{2. ...mais des expériences bénévoles qui témoignent d'une logique d'exclusion sociale}

Cette quête d'une valorisation strictement économique du bénévolat de compétences des seniors peut également être lue à la lumière des pratiques d'éviction précoce des salariés âgés du marché du marché du travail. Elles marquent une rupture brutale conduisant certains seniors à se tourner vers une activité qui fasse écho à la fonction occupée par le passé. Les responsables d'Ecti interviewés n’échappent pas à ce modèle de retraite-couperet. 
L'expérience de Marc en est une illustration. Cet ingénieur de formation a repris, redressé et dirigé des PME pour le compte d'une multinationale pendant 15 années. Mais, à 55 ans, la cession de la filiale qu'il dirige le précipite dans une situation de chômage. Coincé dans le sas de la transition travail-retraite, il s'occupe tant que mal en exerçant des missions ponctuelles de conseil auprès des PME et en délivrant des cours au sein d'une École de commerce. C'est seulement au bout de 6 années, au moment où il commence à toucher sa pension de retraite, que Marc s'engage comme bénévole au sein d'Ecti. Cette implication, précise-t-il, est un moyen de « vivre encore pendant quelques années ce que j'ai vécu en entreprise. J'ai adoré moi être chef d'entreprise ! (...) À Ecti, je peux aussi réutiliser mes compétences et ainsi garder ce qui me rappelle ce moment de ma vie ». Ce sentiment est largement partagé par les responsables interrogés. La plupart qui ont évoqué un chômage en fin de carrière (6/15) n'ont d'ailleurs pas attendu la pension avant de s'engager bénévolement; invoquant, comme Antoine, la difficulté de retrouver un emploi à ces âges avancés : «Je n'étais pas motivé pour retrouver un autre job ou monter ma boîte comme certains de mes amis (...) et de toute façon peu ont réussi à se remettre en selle !».

Ces ruptures professionnelles en fin de carrière ont manifestement conduit nombre de nos responsables à des parcours d'insertion sociale par le bénévolat, à défaut d'une inclusion par la reprise d'une activité marchande. Cette situation aurait pu engendrer leur implication dans des associations de cadres quinquagénaires en recherche d'emploi dont la « cause » a été mise en lumière par Marielle Poussou-Plesse (PoussouPlesse 2007). Mais à l'évidence, nos responsables sont des archétypes de cadres du privé peu enclins à l'action revendicative, contrairement aux autres catégories de salariés. Cela étant, l'exclusion du monde social du travail engendrée par ces retraits précoces semble se reproduire à l'identique dès lors qu'ils intègrent le secteur associatif, du moins là où le bénévolat de compétences est de rigueur. Les pouvoirs publics, les professionnels du conseil en entreprise tout comme les bénéficiaires des missions délivrées par l'association se montrent en effet rétifs à l'égard de ce bénévolat senior. Leurs crispations rappellent que le bénévole peut être considéré comme un outsider au monde du travail, un dissident qui attise les regards soupçonneux (FerrandBechmann 1992 ; Simonet-Cusset 2004). Mais qu'est-il ainsi reproché aux bénévoles seniors d'Ecti ? Ces situations peuvent -elles les conduire à des actions de contestation?

Face aux bénéficiaires de missions, ces bénévoles sont parfois confrontés à des situations de rejet qu'ils mettent sur le compte d'un présupposé négatif à l'égard du travail bénévole. Le témoignage de Michel va dans ce sens.

Le problème c'est que, quand on va vers les entreprises, si on dit trop qu'on est bénévole sans insister sur le côté professionnalisme de la chose, on va dire : "mais vous êtes bénévole, ça veut dire que ce n'est pas sérieux, etc.". Donc on insiste bien sur le fait que nous sommes bénévoles, mais que nous menons nos missions de façon professionnelle. » (Michel, 1er interview)

L'expérience vécue par Sophie dans l'exercice d'une mission est en cela édifiante: 
«Une fois, je suis partie faire une mission en Tunisie pour donner une formation pointue. J'avais vraiment du mal à me faire payer et j'ai été reçue par la secrétaire. Tout ça parce que j'étais bénévole. Pour moi, tout ça c'était incompréhensible, car au départ, ils m'avaient choisie pour ma compétence et j'avais plus d'expériences que les petits jeunes consultants. D'ailleurs quand on fait des missions, le demandeur ne souhaite pas toujours qu'on dise qu'on vient d'Ecti tout simplement, car on ne nous paie pas. » (Sophie, 1er interview)

On s'aperçoit bien vite que cette remise en cause du travail bénévole s'accompagne d'une dévalorisation des compétences des seniors d'Ecti. Comme si la pratique du bénévolat par ces personnes extrêmement dotées en ressources participait, au même titre que la retraite elle-même, à leur exclusion d'une société dominée par l'activité de travail. Ceci révèle l'inadéquation voire l'incompatibilité entre les statuts de bénévole et de professionnel, et la persistance d'une spécialisation des différents temps sociaux qui rend difficile toute tentative de conciliation entre travail et retraite.

\subsection{Puis une association qui se (re)positionne dans la sphère publique}

Ces expériences vécues par les responsables d’Ecti invitent aussi à regarder avec une plus grande lucidité les réticences des entreprises et des pouvoirs publics à l'égard du bénévolat de compétences des seniors. On comprend que les reproches se cristallisent sur la possibilité que ce bénévolat fasse concurrence à l'emploi, au secteur marchand et plus précisément au travail des consultants. Cette lucidité est perceptible dans les propos de Michel qui, lors d'une deuxième interview, témoigne du travail d'introspection auquel se sont livrés les responsables de l'association ces dernières années : «Il y a trois ans, notre positionnement était de dire qu'on a une ambiguïté parce que, sur le marché, les gens qui sont sur ce créneau de l'accompagnement et de l'aide nous voient comme une concurrence déloyale parce que nous sommes gratuits ». C'est ainsi qu'au bout de dix années d'une stratégie de professionnalisation, les difficultés rencontrées pour faire accepter le bénévolat de compétences des seniors conduisent à une révision de l'orientation de l'association.

Sans véritablement remettre en question le principe de professionnalisation de l'action bénévole, les réponses de l'association semblent aujourd'hui davantage porter sur une volonté de rectifier la perception à l'égard des finalités de ce bénévolat. Aux consultants rétifs, Ecti propose un partage de l'espace d'intervention en se cantonnant à une "clientèle » dont la trésorerie ne permet pas de rémunérer un travail d'expertise. «On a positionné notre association beaucoup plus sereinement en disant "nous sommes complémentaires". C'est-à-dire que, oui, on accompagne des PME, des cabinets peuvent le faire, mais des PME ne sont pas financièrement capables de se payer un consultant dans certains cas », indique Michel. Mais une interrogation demeure quant à la réalité objective de ce "deal", ne serait-ce que sur la réelle adhésion des consultants à cette proposition. 
Face aux pouvoirs publics, l'association porte le même discours qu'auprès des consultants, mais, surtout, s'inscrit dans une démarche d'action collective. Ces dernières années, les faisceaux d'action se sont en effet progressivement tournés vers une logique de réseau avec la création, en octobre 2012, d'un collectif dénommé " groupe de travail sur l'engagement bénévole des seniors ». Ce groupe fédère, entre autres, les trois organisations nationales qui promeuvent le bénévolat de compétences des seniors: les associations Ecti, EGEE (Entente des générations pour l'emploi et l'entreprise et AGIR-ABCD (Association générale des intervenants retraités - Action de bénévoles pour la coopération et le développement). Pour Michel, le porteur du projet au sein d'Ecti, l'objectif est « d'inciter les pouvoirs publics à mieux utiliser nos forces. Notre approche est de les sensibiliser à cette force qu'on a et qui permet aussi, parce que nous ne sommes pas dans du caritatif, de leur dire "nous pouvons être très utiles au maintien et à la création de l'emploi parce que nous travaillons vers les créateurs d'entreprise, nous aidons les TPE et les PME à s'en sortir par nos activités d'assistance, de conseil et d'accompagnement. "

L'originalité de ce collectif tient à l'opportunité qu'il saisit de transformer en «lobby » une somme de frustrations nées des suspicions à l'égard du bénévolat d'expertise et des restrictions d'espaces et de catégories d'actions qui en découlent. Partant du constat qu'« aucune mesure concrète ne semble s'intéresser au rôle des seniors dans la société, à leurs atouts et aux enjeux d'une bonne transition entre vie active et retraite », ce collectif se propose d'examiner et de produire des rapports d'études destinés aux pouvoirs publics et aux entreprises sur « les dispositifs en place ou en cours d'élaboration, se rapportant notamment à la transition vers la retraite, à la sensibilisation à l'engagement associatif et à la transmission intergénérationnelle ».

\section{Conclusion}

Notre cheminement nous a conduits à comprendre le bénévolat de compétences des seniors à la lumière des dynamiques de professionnalisation de l'activité bénévole au sein de l'association Ecti. Si au premier regard, cette forme de bénévolat apparaît comme une quête de valorisation économique de l'activité bénévole, cette finalité n'est pas nécessairement partagée par l'ensemble des individus impliqués dans l'action. L'association apparaît plus comme le refuge d'une catégorie de cadres enclins à la reconquête d'un rôle et d'un statut social perdus, et moins comme le lieu où se forme et se cristallise un mouvement de revendication portant les intérêts d'un groupe d'âge particulier.

8 Source : communiqué de presse du 11 octobre 2012 annonçant la création du « Groupe de travail sur l'engagement bénévole des seniors ». 
Mais c'est sans compter les soupçons de concurrence déloyale vis-à-vis de certaines catégories d'actifs qui pèsent sur les bénévoles seniors autant que sur l'image de l'association. C'est parce qu'elles contribuent à dévaloriser encore une fois les ressources des seniors et à leur dénier le statut de bénévole que ces suspicions à l'égard du bénévolat de compétences des seniors ont produit un double mouvement de résistance amorcé par l'association.

Elles l'ont d'abord conduite à se positionner dans un entre-deux, une zone intermédiaire où l'association prend de la distance avec une valorisation exclusivement marchande de l'activité bénévole. Une prise de recul qui se fait au profit d'une activité dont l'association tire une reconnaissance du fait de son utilité sociale. Mais ensuite, ces suspicions ont poussé l'association à s'impliquer dans un collectif d'organisations qui se veut être un groupe de pression porteur des intérêts des seniors auprès des instances de prise décisions tant publiques que privées. L'originalité de ce collectif tient surtout à la configuration du groupe d'âge au nom duquel il se prononce. En se positionnant comme une instance qui représente simultanément les intérêts des travailleurs âgés et des retraités, ce collectif s'affranchit des clivages qui attribuent aux individus, en fonction de leur âge, des rôles, des statuts sociaux et donc des intérêts différents.

En mettant bout à bout les mouvements (internes et externes) amorcés par l'association Ecti, on peut avancer que le bénévolat de compétences des seniors pourrait être à l'origine d'un modèle de revendication qui réclame le droit à l'activité, peu importe sa forme (travail ou bénévolat) ou sa finalité (marchande ou non marchande). Cette forme de "retraite-conciliation " rejoindrait alors une approche du type « vieillissement productif » qui justifie le rôle des retraités par le bénéfice que la société tire de leurs activités non marchandes. Elle serait aussi susceptible de rentrer en conflit avec le modèle dominant qui structure la société autour de la centralité du travail. Mais la portée et la valeur heuristique de ce modèle de « retraite-conciliation » doivent être questionnées quand on sait qu'il a été construit en prenant appui sur la catégorie socioprofessionnelle limitée des cadres.

\section{Bibliographie}

Archambault E. (2005) : «Le bénévolat en France et en Europe », Pensée plurielle, vol. $1 / 2005, n^{\circ} 9$, p. 11-34.

Baculard O. (2006) : " Bénévolat de compétences, une nouvelle forme de mécénat ». Volonteer. Paris : France Bénévolat.

Bernardeau-Moreau D., Hély M. (2007) : «Transformations et inerties du bénévolat associatif sur la période 1982-2002». Sociologies pratiques, vol. 2007/2, n 15, pp. 9-

23. 
Bory A. (2013) : « Le bénévolat d'entreprise en France ». Travail et emploi, nº 133, pp. 5362.

Ferrand-Bechman D. (1992) : Bénévolat et solidarité. Paris : Syros-Alternatives.

Percheron A., Rémond R. (dir.) (1991) : Âge et politique. Paris : Economica.

Godbout J.-T. (1994) : « La sphère du don entre étrangers : le bénévolat et l'entraide ». In F. Dumont, S. Langlois, Y. Martin (dir.) Traité des problèmes sociaux, Québec: Institut québécois de recherche sur la culture, pp. 981-994.

Guillemard A-M. (2010): Les défis du vieillissement. Âge, emploi, retraite, perspectives internationales. Paris : Armand Colin.

Guillemard A-M. (1972) : La retraite, une mort sociale. Paris : Mouton.

Laville J.-L. (2003) : « La pluralité des ressources humaines dans les associations ». In J. Allouche (coord.), Encyclopédie des ressources humaines. Paris : Vuibert, pp. 83-91.

Piris F., Dupuy R. (2007): " Mobilisation de ressources dans l'expression de la compétence en activité ». Les cahiers internationaux de psychologie sociale, vol. 2/2007, n 74 , pp. 67-89.

Poussou-Plesse M. (2007): " Du principe d'une "seconde partie de carrière" à la défense d'une cause des “quinquas”. In D. Reguer (dir.), Vieillissement et parcours de fins de carrière : contraintes et stratégies. Paris : ERES, pp. 125-156.

Simonet-Cusset M. (2004) : "Penser le bénévolat comme travail pour repenser la sociologie du travail ». In D. Ferrand-Bechmann (dir.) Les bénévoles et leurs associations. Autres réalités, autre sociologie?, Paris : L’Harmattan, pp. 247-261.

Viriot Durandal J.P., Reguer D. (2011) : « Retraite, engagement social et citoyenneté ». Gérontologie et société, $\mathrm{n}^{\circ}$ 138, pp. 143-164.

Viriot Durandal J.-P. (2003) : Le pouvoir gris. Sociologie des groupes de pression de retraités. Paris : PUF.

Zarifian P. (2001) : Objectif compétence. Pour une nouvelle logique. Paris: Éditions Liaisons (1ère éd., 1999). 